\title{
Augmentation of Hypoxic Pulmonary Vasoconstriction in the Isolated Perfused Rat Lung by In Vitro Antagonists of Endothelium-dependent Relaxation
}

\author{
Valentina L. Brashers, Michael J. Peach, and C. Edward Rose, Jr. \\ Departments of Internal Medicine and Pharmacology, University of Virginia School of Medicine, Charlottesville, Virginia 22908
}

\begin{abstract}
The role of the endothelium in hypoxic constriction of the intact pulmonary vascular bed has not been clearly elucidated. To test for a possible role for endothelium-derived relaxing factor(s) (EDRF) in the hypoxic pressor response, isolated, whole blood-perfused rat lungs from male Sprague-Dawley rats treated with meclofenamate were prepared. Three protocols were performed, including: (a) normal saline (control); (b) the putative EDRF inhibitors, eicosatetraynoic acid (ETYA, 1 $\times 10^{-4} \mathrm{M}$ ) or nordihydroguaiaretic acid (NDGA, $1 \times 10^{-4} \mathrm{M}$ ) versus vehicle DMSO; and (c) the putative EDRF inhibitor hydroquinone (HQ, $1 \times 10^{-4} \mathrm{M}$ ) versus vehicle ethyl alcohol (ETOH). The pulmonary pressor response to angiotensin II (Ang II, $0.25 \mu \mathrm{g}$ ) injections alternated with 6-min periods of hypoxic ventilation $\left(3 \% \mathrm{O}_{2}, 5 \% \mathrm{CO}_{2}\right)$ was measured before and after the administration of saline, inhibitors, or vehicles. The administration of the EDRF inhibitors ETYA, NDGA, and HQ resulted in a marked accentuation of the hypoxic pressor response that was not seen in the controls $(P<0.05)$. In separate experiments, lungs precontracted with norepinephrine (1 $\left.\times 10^{-6} \mathrm{M}\right)$ were pretreated with edrophonium $\left(1 \times 10^{-4} \mathrm{M}\right)$ and then observed for endothelium-dependent vasodilator responses to acetylcholine at increasing doses $\left(1 \times 10^{-7}-1\right.$ $\times 10^{-4} \mathrm{M}$ ). Administration of ETYA, NDGA, or HQ abrogated the observed vasodilatation to acetylcholine, which was not seen with vehicles alone $(P<0.01)$. These studies suggest an important role for the endothelium in pulmonary vascular responsiveness to alveolar hypoxia through possible release of a relaxing factor(s) that attenuates the degree of pulmonary arterial constriction.
\end{abstract}

\section{Introduction}

Hypoxic pulmonary hypertension remains a major cause of morbidity and mortality in patients with progressive pulmonary disease (1). Despite the initial description of hypoxic pulmonary arterial constriction in 1946 (2), the mechanism(s) responsible for modulation of vascular smooth muscle contraction have not been clearly elucidated. In 1980, Furchgott and Zawadzki (3) reported that an intact endothelium was important for the relaxation of precontracted vascular rings to acetylcholine, as rings denuded of endothelium failed to vaso-

Address reprint requests to Dr. C. Edward Rose, Jr., Box 225 Pulmonary Division, University of Virginia School of Medicine, Charlottesville, VA 22908. 1988.

Received for publication 13 July 1987 and in revised form 1 June

J. Clin. Invest.

(c) The American Society for Clinical Investigation, Inc.

$0021-9738 / 88 / 11 / 1495 / 08 \quad \$ 2.00$

Volume 82, November 1988, 1495-1502 dilate to acetylcholine. Subsequent studies demonstrated that this endothelium-dependent vasodilation existed for some (acetylcholine, bradykinin, ATP, and AA, etc.) but not all (nitroglycerin, nitroprusside, isoproterenol, and adenosine) vasodilators $(4,5)$. As the role of the endothelium was investigated further, it was apparent that endothelium-dependent relaxation was due to release of a diffusible factor(s), as the relaxation was transferable in perfusate to vascular strips denuded of endothelium (6). This factor appeared to be a nonprostaglandin substance since vascular responsiveness to endothelium-dependent vasodilators was unchanged by treatment with cyclooxygenase inhibitors $(7,8)$. However, the actions of these endothelium-dependent relaxing factors (EDRF) ${ }^{1}$ were attenuated or blocked by multiple pharmacological agents, including the lipoxygenase antagonists eicosatetrayenoic acid (ETYA) and nordihydroguaiaretic acid (NDGA) (8) and the antioxidant hydroquinone (HQ) (6).

In addition to the evidence for a role of the endothelium in vascular responses to certain vasodilators in these in vitro studies, recent work has demonstrated a possible role for EDRF in the intact pulmonary circulation (9). However, involvement of EDRF in hypoxic pulmonary vasoconstriction has never been characterized. The purpose of this study was to test for a possible role of the endothelium in pulmonary vascular responses to alveolar hypoxia and angiotensin II in the intact pulmonary vascular bed using the isolated perfused lung preparation.

\section{Methods}

\section{Isolated perfused lung}

The isolated perfused lung preparation originally described by Hauge (10) and modified by McMurtry et al. (11) was used to evaluate the hypoxic pulmonary pressor response. Male Sprague-Dawley rats weighing 300-450 g were anesthetized with $30 \mathrm{mg} / \mathrm{kg}$ i.p. pentobarbital sodium (Vet Labs Limited, Inc., Lenexa, KS). After tracheal cannulation, the lungs were ventilated (model 361; Harvard Apparatus Co., S. Natick, MA) with warmed normoxic gas $\left(95 \%\right.$ air, $\left.5 \% \mathrm{CO}_{2}\right)$ at a rate of 60 breaths per minute and $2 \mathrm{mmHg}$ positive end-expiratory pressure. Tidal volume was adjusted to maintain a peak inspiratory pressure of $10 \mathrm{mmHg}$. A median sternotomy was performed and $100 \mathrm{U}(0.1 \mathrm{ml})$ of heparin (Elkins-Sinn, Inc., Cherry Hill, NJ) was injected into the right ventricle. Cannulae were inserted into the pulmonary artery and left ventricle. The heart and lungs were excised carefully and suspended in a humidified chamber at $38^{\circ} \mathrm{C}$. The isolated lung preparation was then perfused with whole blood warmed to $38^{\circ} \mathrm{C}$ at $0.03 \mathrm{ml} / \mathrm{g}$ body weight per min, using a peristaltic pump (Critikon, Inc., Tampa, FL). 25-30 $\mathrm{ml}$ of whole blood for each experiment was obtained from cardiac puncture of male Sprague-Dawley blood-donor rats weighing $>500 \mathrm{~g}$ in preheparinized syringes, after deep anesthesia by diethyl ether inhalation (Fisher Scientific Company, Fair Lawn, NJ). This blood was filtered through nylon mesh into a reservoir surrounded by a water

1. Abbreviations used in this paper: Ang II, angiotensin II; EDRF, endothelium-dependent relaxing factor; ETOH, ethyl alcohol; ETYA, eicosatetraynoic acid; HG, hydroquinone; Hypox, hypoxia; NDGA, nordihydroguaieretic acid; NE, norepinephrine. 
jacket with circulated water at $38^{\circ} \mathrm{C}$. Blood was warmed to $38^{\circ} \mathrm{C}$ before entering the pulmonary artery by the reservoir and by passing through a perfusion coil bathed by water at $38^{\circ} \mathrm{C}$ using a circulating water bath (Haake, Inc., Karlsruhe, FRG). Effluent blood from the left ventricle was returned to the reservoir for recirculation. Throughout the experiment, blood $\mathrm{pH}$ was maintained between 7.30 and 7.50 by addition of $\mathrm{NaHCO}_{3}$ (American Hospital Division, Shirley, NJ) to the blood reservoir.

\section{Measurements}

Pulmonary arterial pressure was measured by a vascular transducer (P23Db; Gould Inc., Cleveland, $\mathrm{OH}$ ) connected to the proximal pulmonary artery tubing, and a strip-chart record (7754B; Hewlett-Packard Co., Palo Alto, CA). Because perfusion rate was constant, increases in pulmonary arterial pressure reflected increase in pulmonary vascular resistance. Airway pressure was measured using a transducer (MP45-1-871; Validyne Engineering Corp., Northridge, CA) connected to the inspiratory tubing from the ventilator. Effluent blood $\mathrm{pH}$, $\mathrm{PO}_{2}$, and $\mathrm{PCO}_{2}$ were measured using appropriate electrodes (model 127; Instrumentation Laboratory, Lexington, MA).

\section{Protocols}

After institution of perfusion, stable baseline pulmonary pressures were established over at least $30 \mathrm{~min}$. Absolute pulmonary artery pressures before the beginning of each experiment were between 10 and $17.5 \mathrm{mmHg}$, and increased at a rate $<5 \mathrm{mmHg} / \mathrm{h}$ during the protocols (Table I). The lungs were then challenged during normoxic ventilation with $0.25 \mu \mathrm{g} \mathrm{Asn}^{1}, \mathrm{Val}^{5}$ angiotensin II (Ang II; CIBA Pharmaceuticals, CIBA-Geigy Corp., Summit, NJ), in $0.05 \mathrm{ml}$ of $\mathrm{NaCl}$, which was injected into the pulmonary arterial tubing just proximal to the cannula. This was followed in 5 min by a 6-min period of ventilation with hypoxic gas (Hypox I; $3 \% \mathrm{O}_{2}, 5 \% \mathrm{CO}_{2}, 92 \% \mathrm{~N}_{2}$ ) to assess the initial reactiveness of the lung vasculature. Any preparation not achieving at least a $5-\mathrm{mmHg}$ increase in pulmonary arterial pressure with this hypoxic challenge was excluded from the study. $5 \mathrm{~min}$ after Hypox I, Ang II was again administered. 5 min later, in all protocols, $20 \mu \mathrm{g} / \mathrm{ml}$ sodium meclofenamate (Warner-Lambert Co., Ann Arbor, MI) in 0.1 $\mathrm{ml}$ of $0.9 \% \mathrm{NaCl}$ was added to the effluent blood returning to the reservoir to eliminate the possibility that effects of other inhibitors subsequently administered were due to cyclooxygenase inhibition or to shunting of the arachidonate cascade to the cyclooxygenase pathway. 5 min after the administration of meclofenamate, the lungs were challenged with three periods of alternating injection of $0.25 \mu \mathrm{g}$ Ang II via the pulmonary artery (Ang II-2 to Ang II-4) and 6 min of hypoxic ventilation (Hypox II to Hypox IV), each separated by 5-min intervals for recovery. Effluent blood $\mathrm{pH}$ and gas tensions were $\mathrm{pH} 7.38 \pm 0.004$, $\mathrm{PO}_{2} 120 \pm 2 \mathrm{mmHg}$, and $\mathrm{PCO}_{2} 31 \pm 1 \mathrm{mmHg}$ during normoxia, and $\mathrm{pH}$ $7.41 \pm 0.002, \mathrm{PO}_{2} 29 \pm 0.4 \mathrm{mmHg}$, and $\mathrm{PCO}_{2} 28 \pm 0.4 \mathrm{mmHg}$ during the fourth minute of hypoxia (mean $\pm \mathrm{SE}, n=48$ ). Vascular reactivity to hypoxia in the isolated perfused rat lung has been previously observed to increase progressively from the first through the fourth hypoxic challenge and then plateau $(11,12)$. To minimize the effect of this change in reactivity, antagonists and their vehicles were administered after Hypox IV. Therefore, 5 min after Hypox IV, vehicles or chemically dissimilar putative antagonists of EDRF were added to the reservoir blood. $5 \mathrm{~min}$ thereafter, four periods of alternating $0.25 \mu \mathrm{g}$ Ang II (Ang II-5 to Ang II-8) via the pulmonary artery, and hypoxic ventilation for 6-min periods (Hypox V to Hypox VIII) were evaluated for changes in pulmonary vascular reactivity. The following protocols were performed by administration of vehicles or antagonists between Hypox IV and Hypox V:

(a) Normal saline alone $(n=8)$. To evaluate for changes in reactivity of our preparation and to control for the effects of vehicles, a separate set of experiments was performed in which $0.05 \mathrm{ml} 0.9 \% \mathrm{NaCl}$ was injected into the reservoir blood between Hypox IV and Hypox $\mathrm{V}$.

(b) ETYA or NDGA $(n=8)$. Both $1 \times 10^{-4} \mathrm{M}$ ETYA and $1 \times 10^{-4}$ $M$ NDGA have been shown to antagonize endothelium-dependent relaxation responses to acetylcholine in precontacted rabbit thoracic aortic segments (8). Therefore, 5,8,11,14-ETYA (Ro3-1428; Hoffmann-La Roche, Inc., Nutley, NJ) in $0.05 \mathrm{ml}$ of DMSO (Fisher Scientific Co.) was added to the reservoir blood for an ETYA final concentration of $1 \times 10^{-4} \mathrm{M}$ between Hypox IV and Hypox $\mathrm{V}$ and remained for the duration of the experimental protocol. In a separate protocol, NDGA (Sigma Chemical Co., St. Louis, MO) in $0.05 \mathrm{ml}$ DMSO was added to the reservoir blood for a reservoir NDGA concentration of 1 $\times 10^{-4} \mathrm{M}$, between Hypox IV and Hypox $\mathrm{V}(n=8)$ and was present through the rest of the protocol. An additional separate group of studies was performed in which the vehicle DMSO $(0.05 \mathrm{ml})$ was added to the reservoir blood between Hypox IV and Hypox $\mathrm{V}$ to determine the effects of the vehicle alone.

(c) Hydroquinone (HQ) $(n=8)$. Previous investigators (5) have observed that the antioxidant 1,4-benzenediol $\left(1 \times 10^{-4} \mathrm{M} \mathrm{HQ}\right)$ abolished relaxation of vascular rings to acetylcholine. Therefore, HQ (Sigma Chemical Co.) in $0.05 \mathrm{ml}$ ethyl alcohol (ETOH; U.S. Industrial Chemicals Co., Tuscola, IL), was added to the reservoir blood for an HQ concentration of $1 \times 10^{-4} \mathrm{M}$ between Hypox IV and Hypox $\mathrm{V}$ and was present through the rest of the protocol. A separate group of studies was performed in which the vehicle ETOH $(0.05 \mathrm{ml})$ was added to the reservoir blood between Hypox IV and Hypox V to observe the effects of vehicle alone.

Efficacy of inhibitors to block endothelium-dependent vasodilators. To assess whether these EDRF inhibitors blocked a known endothelium-dependent vasodilatory response in the isolated perfused rat lung, studies were performed in a separate group of isolated blood-perfused lungs prepared as described above. Initial responsiveness of the pulmonary vascular bed to Ang II and hypoxia was established, after which vascular resistance was elevated by the addition of norepinephrine (NE; Sigma Chemical Co.) in $0.05 \mathrm{ml} 0.9 \% \mathrm{NaCl}$ to result in a reservoir blood concentration of $1 \times 10^{-6} \mathrm{M} .2 \mathrm{~min}$ after the NE following peak pulmonary vasoconstriction, edrophonium chloride (Sigma Chemical Co.) was administered in $0.05 \mathrm{ml} 0.9 \% \mathrm{NaCl}$ to attain a reservoir blood concentration of $1 \times 10^{-4} \mathrm{M}$. Edrophonium was administered to block the acetylcholine esterase activity of red blood cells and the vasculature, which could obscure vascular responses to acetylcholine (13). 2 min later, the endothelium-dependent vasodilator acetylcholine chloride (Sigma Chemical Co.) was administered in $0.05 \mathrm{ml} 0.9 \% \mathrm{NaCl}$ in increasing doses at 1 -min intervals into the pulmonary artery to achieve total reservoir blood acetylcholine concentrations of $1 \times 10^{-7}$, $1 \times 10^{-6}, 1 \times 10^{-5}$, and $1 \times 10^{-4} \mathrm{M}$. Acetylcholine solutions were prepared by thawing and diluting previously prepared stock solutions of acetylcholine in $0.9 \%$ normal saline. After $10 \mathrm{~min}$ for recovery, either ETYA $\left(1 \times 10^{-4} \mathrm{M}, n=6\right)$, NDGA $\left(1 \times 10^{-4} \mathrm{M}, n=6\right)$, or HQ $\left(1 \times 10^{-4} \mathrm{M}, n=6\right)$ was added to the reservoir blood, followed in $5 \mathrm{~min}$ by identical administration of $1 \times 10^{-4} \mathrm{M}$ edrophonium into the reservoir blood. Acetylcholine was injected again in identical doses into the pulmonary artery at 1-min intervals. A separate group of experiments was performed in lungs treated as above except that either vehicle DMSO $(0.05 \mathrm{ml}, n=6)$ or vehicle ETOH, $(0.05 \mathrm{ml}, n=6)$ was added to the reservoir blood rather than inhibitors to determine the effects of these vehicles alone.

Statistical analysis. The rise in pulmonary artery pressure $(\triangle \mathrm{PA})$ with Ang II and hypoxic ventilation was calculated for each of eight periods in each protocol. Changes in the $\triangle \mathrm{PA}$ within each protocol were identified by one-way analysis of variance and Student-Newman-Keuls' multiple comparisons test (14). $T$ statistics calculated from the least-square means and pooled variance adjusted for subject-specific period IV pulmonary arterial pressure changes were performed to evaluate changes in $\triangle P A$ between vehicles and inhibitors. Statistical significance was identified when $P<0.05$. In the text, tables, and figures, data are expressed as mean $\pm \mathrm{SE}$.

\section{Results}

Effects of normal saline (Table I, Fig. 1). Baseline pulmonary artery pressure was stable and increased at a rate of $<5$ 
Table I. Change in Baseline Pulmonary Arterial Pressure

\begin{tabular}{lcc}
\hline \multicolumn{1}{c}{ Protocol } & $\begin{array}{c}\text { Baseline pulmonary } \\
\text { artery pressure before } \\
\text { Hypox I }\end{array}$ & $\begin{array}{c}\text { Rate of rise in pulmonary } \\
\text { artery pressure during } \\
\text { protocol }\end{array}$ \\
\hline Normal saline & $m m H g$ & $m m H g / h$ \\
ETYA & $15 \pm 1$ & $2 \pm 1$ \\
NDGA & $18 \pm 1$ & $2 \pm 1$ \\
DMSO & $14 \pm 1$ & $4 \pm 1$ \\
HQ & $16 \pm 1$ & $3 \pm 1$ \\
ETOH & $16 \pm 1$ & $5 \pm 1$ \\
\hline
\end{tabular}

$\mathrm{mmHg} / \mathrm{h}$ during the various treatment and vehicle protocols (Table I). The rise in pulmonary arterial pressure with hypoxia was stable with repeated hypoxic challenges (Fig. 1). Addition of normal saline to the reservoir blood between the fourth and fifth hypoxic challenges failed to alter the rise in pulmonary artery pressure induced by hypoxia. The $\triangle \mathrm{PA}$ in response to Ang II was increased after meclofenamate administration (period II compared with period I, $P<0.01$ ) in this and in all subsequent protocols (Fig. 1).

Effect of ETYA or NDGA (Figs. 2-4). Baseline pulmonary artery pressure before all hypoxic challenges were comparable among the ETYA, NDGA, and DMSO protocols and rose at a rate of $<4 \mathrm{mmHg} / \mathrm{h}$ in all groups (Table I). There was no significant difference in the hypoxic pressor response between any of the protocols during the first four hypoxic challenges. Administration of the vehicle DMSO to the reservoir blood between the fourth and fifth hypoxic challenge resulted in no significant change in the $\triangle \mathrm{PA}$ response to hypoxic ventilation (Figs. 2 and 3). In striking contrast, the addition of either ETYA (Fig. 2) or NDGA (Fig. 3) to the reservoir blood between Hypox IV and Hypox $\mathrm{V}$ resulted in a marked accentuation $(P<0.05)$ of the hypoxic pressor response that persisted through the eighth period, which was significantly different ( $P$ $<0.05$ ) from the response obtained with DMSO alone. In contrast to the hypoxic pressor response, the rise in pulmonary arterial pressure to $0.25 \mu \mathrm{g}$ Ang II was augmented by administration of DMSO (Fig. 4). Comparable increases in the pressor responses to Ang II were observed after administration of either ETYA or NDGA (Fig. 4).

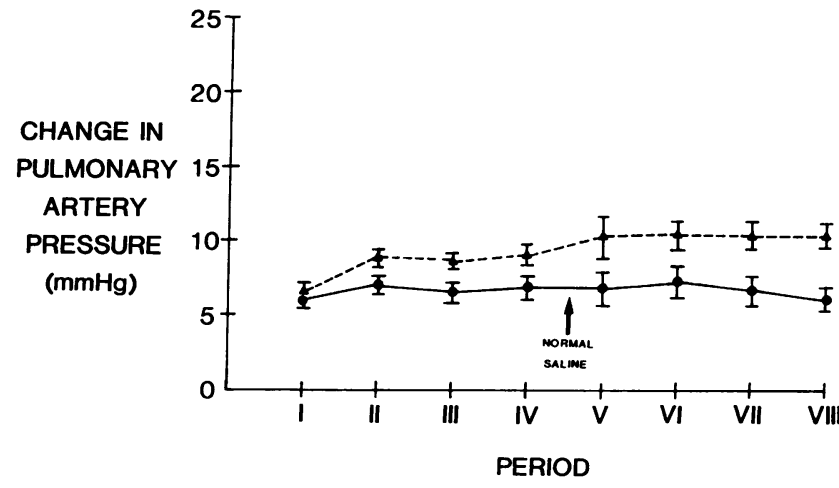

Figure 1. Administration of normal saline between Hypox IV and Hypox $\mathrm{V}$ was not associated with any change in the hypoxic pressor response in the subsequent periods. Pulmonary arterial pressure responses to Ang II injection remained stable during the protocol. $\boldsymbol{n}=\mathbf{8}$. •, hypoxic ventilation; $\Delta$, Ang II injection.

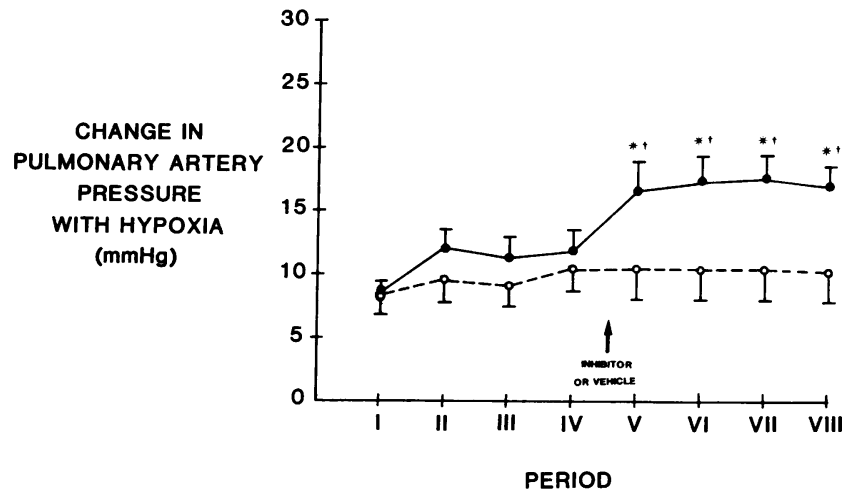

Figure 2. The administration of $1 \times 10^{-4} \mathrm{M}$ ETYA between Hypox IV and Hypox $\mathrm{V}$ resulted in a marked accentuation of the hypoxic pressor response in subsequent periods, as compared with the vehicle DMSO. $n=8$. ๑, ETYA, O, DMSO. *Significantly different from periods III and IV, $P<0.05$. 'Significant difference between the change in response from period IV to the subsequent period after ETYA compared with the change between comparable periods with DMSO, $P<0.05$.

Effects of $H Q$ (Figs. 5 and 6). There was no significant difference in the absolute pulmonary artery pressure before each hypoxic challenge between the studies using HQ or its vehicle, ethyl alcohol. Baseline pulmonary arterial pressure increased at a rate of $\leq 5 \mathrm{mmHg} / \mathrm{h}$ in both groups (Table I). Administration of vehicle to the reservoir blood between Hypox IV and Hypox V resulted in no significant increase in the hypoxic pressor response (Fig. 5). However, the addition of HQ to the reservoir blood between the fourth and fifth hypoxic challenges resulted in a marked potentiation $(P<0.05)$ of the hypoxic pulmonary vasoconstrictor response that persisted through the eighth period (Fig. 5). This change in $\triangle \mathrm{PA}$ with hypoxia was significantly greater $(P<0.05)$ after the administration of HQ as compared to the vehicle ETOH alone. The pulmonary pressor response to Ang II increased $(P<0.05)$ comparably in both protocols after the administration of HQ or vehicle to the reservoir blood between the fourth and fifth periods (Fig. 6).

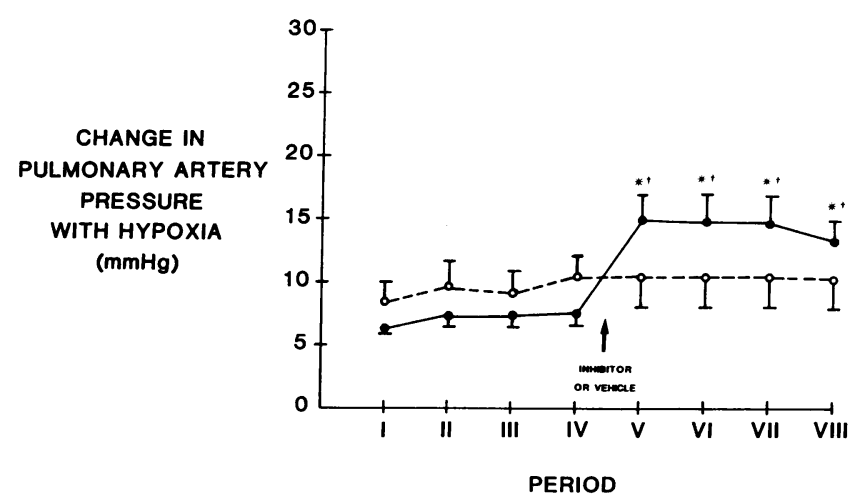

Figure 3. The administration of $1 \times 10^{-4} \mathrm{M}$ NDGA between Hypox IV and Hypox $\mathrm{V}$ markedly potentiated the hypoxic pressor response in subsequent periods as compared with the vehicle DMSO. $n=8$. $\bullet$, NDGA, O, DMSO. *Significantly different from periods III and IV, $P$ $<0.05$. 'Significant difference between the change in response from period IV to the subsequent period with NDGA compared with the change between comparable periods with DMSO, $P<0.05$. 


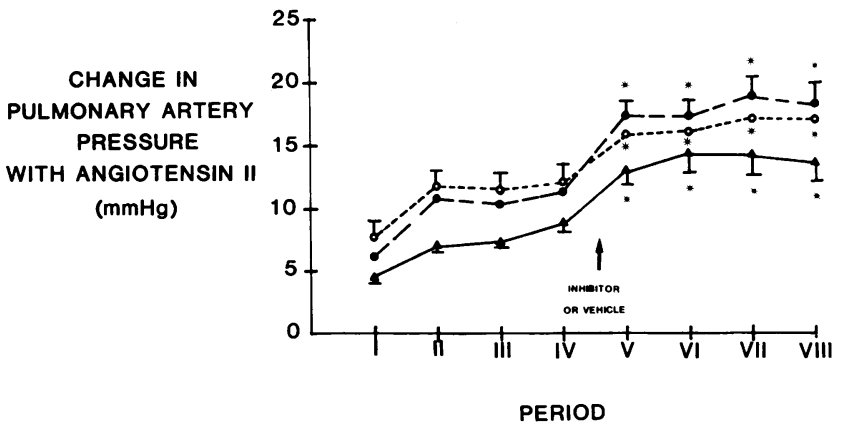

Figure 4. The pulmonary arterial pressor response to Ang II injection increased equivalently in all protocols after the administration of either $1 \times 10^{-4} \mathrm{M}$ ETYA, $1 \times 10^{-4} \mathrm{M}$ NDGA, or DMSO between periods IV and V. $n=8$. $\bullet$, ETYA, $\triangle$, NDGA, and $\circ$, DMSO. *Significantly different from periods III and IV, $P<0.05$.

Efficacy of inhibitors to block endothelium-dependent vasodilators (Figs. 7 and 8). Pulmonary arterial administration of acetylcholine (Figs. 7 and 8) resulted in a dose-dependent relaxation of the NE-precontracted pulmonary vasculature after pretreatment with edrophonium in all studies before the addition of ETYA, NDGA, or HQ or the vehicles DMSO or ETOH to the reservoir blood $(P<0.05)$. The addition of the vehicles DMSO or ETOH resulted in no significant change in this acetylcholine-induced pulmonary arterial vasodilation (Figs. $7 A$ and $8 A$ ). Administration of ETYA, NDGA, or HQ to the reservoir blood completely abolished the relaxation response to acetylcholine and instead a mild increase $(P<0.05)$ in pulmonary arterial pressure was observed (Figs. 7, $B$ and $C$, and Fig. $8 \mathrm{~B}$ ). The changes in pulmonary arterial pressure responses to acetylcholine after ETYA, NDGA, or HQ administration were significantly different from those seen after vehicle administration.

\section{Discussion}

Although the pulmonary arterial constrictor response to alveolar hypoxia has been investigated extensively, its precise

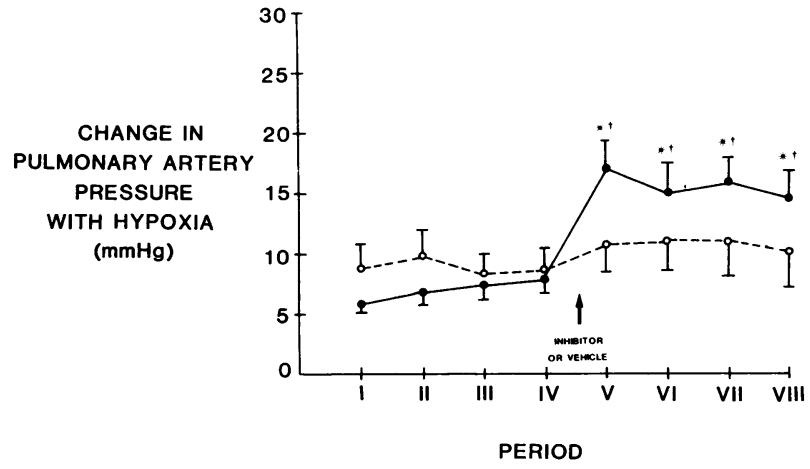

Figure 5. The administration of $1 \times 10^{-4} \mathrm{M}$ HQ between Hypox IV and Hypox $\mathrm{V}$ resulted in a marked accentuation of the hypoxic pressor response in subsequent periods as compared with ETOH vehicle control. $n=8$. •, HQ; O, ETOH. *Significantly different from period III and IV, $P<0.05$. 'Significant difference between the change in response from period IV to the subsequent period after HQ compared with the change between comparable periods with ETOH, $P<0.05$.

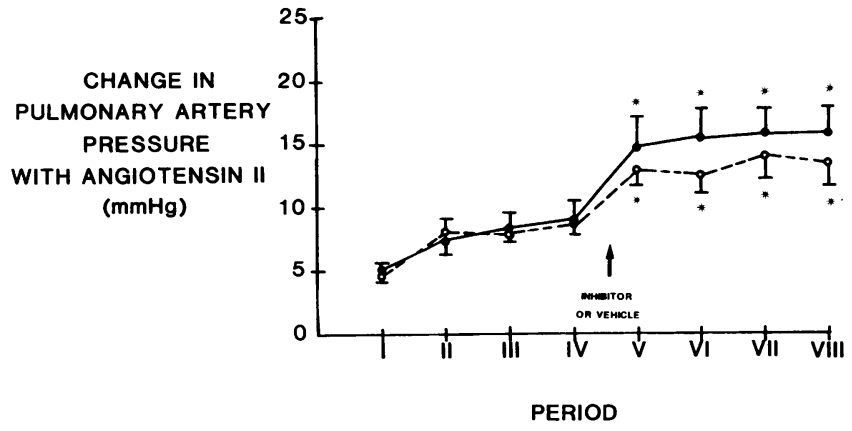

Figure 6. The pulmonary arterial pressor response to Ang II injection increased comparably in both protocols after the administration of 1 $\times 10^{-4} \mathrm{M}$ HQ or ETOH between periods IV and V. $n=8$. $\bullet$, HQ; $O$, ETOH. *Significantly different from periods III and IV, $P<0.05$.

mechanism and factors that modulate the vasoconstriction have not been clearly explained. This study demonstrated that the chemically dissimilar EDRF inhibitors ETYA, NDGA, and $H Q$ all augmented the hypoxia-induced $\triangle \mathrm{PA}$ in the denervated but otherwise intact pulmonary circulation of the isolated perfused rat lung. In addition, pulmonary vasodilation was observed with administration of the endothelium-dependent vasodilator acetylcholme, and this vasodilation was abolished by pretreatment with the EDRF inhibitors ETYA, NDGA, and HQ.

These studies were conducted using an isolated, denervated lung preparation and suggest that the endothelium is an important modulator of the hypoxic pressor response. Pulmonary arterial constriction has been shown to occur in response to alveolar hypoxia in vivo with ventilation of one lung with hypoxic gas (15). Furthermore, isolated, denervated, salineperfused rat lungs have exhibited continued pressor responses to hypoxic ventilation (11). These observations imply that the sensors and effectors for hypoxic pulmonary vasoconstriction function locally for control of blood flow, and the response is independent of substantial influence from the autonomic nervous system or systemic humoral substances (16). Although some investigators have suggested that pulmonary arterial constriction is due to direct effects of hypoxia on vascular smooth muscle cellular membrane ion transport mechanisms (17) and excitation-contraction coupling (11), recent studies have emphasized the importance of the endothelium in mediating vascular responsiveness to hypoxia. DeMey and Vanhoutte (18) observed that augmentation by anoxia of canine femoral artery strip contraction to NE was enhanced by the presence of an intact endothelium; they went on to document the same finding in pulmonary artery strips (19). Peach et al. (5) found that anoxia produced an endothelium-dependent vasoconstriction in rabbit and rat aorta. Holden and McCall (20) showed that an intact endothelium was necessary for hypoxia-induced contractions of in vitro main pulmonary artery strips from pigs. The question that is raised by these investigations is whether hypoxia causes the release of a vasoconstrictor substance $(21,22)$ or rather interrupts the production, release, and/or action of a vasodilator substance from the endothelium that exerts modulating effects on vascular smooth muscle responses to hypoxia.

Since Furchgott and Zawadzki (3) first demonstrated that rat aortic strips would relax in response to acetylcholine only 


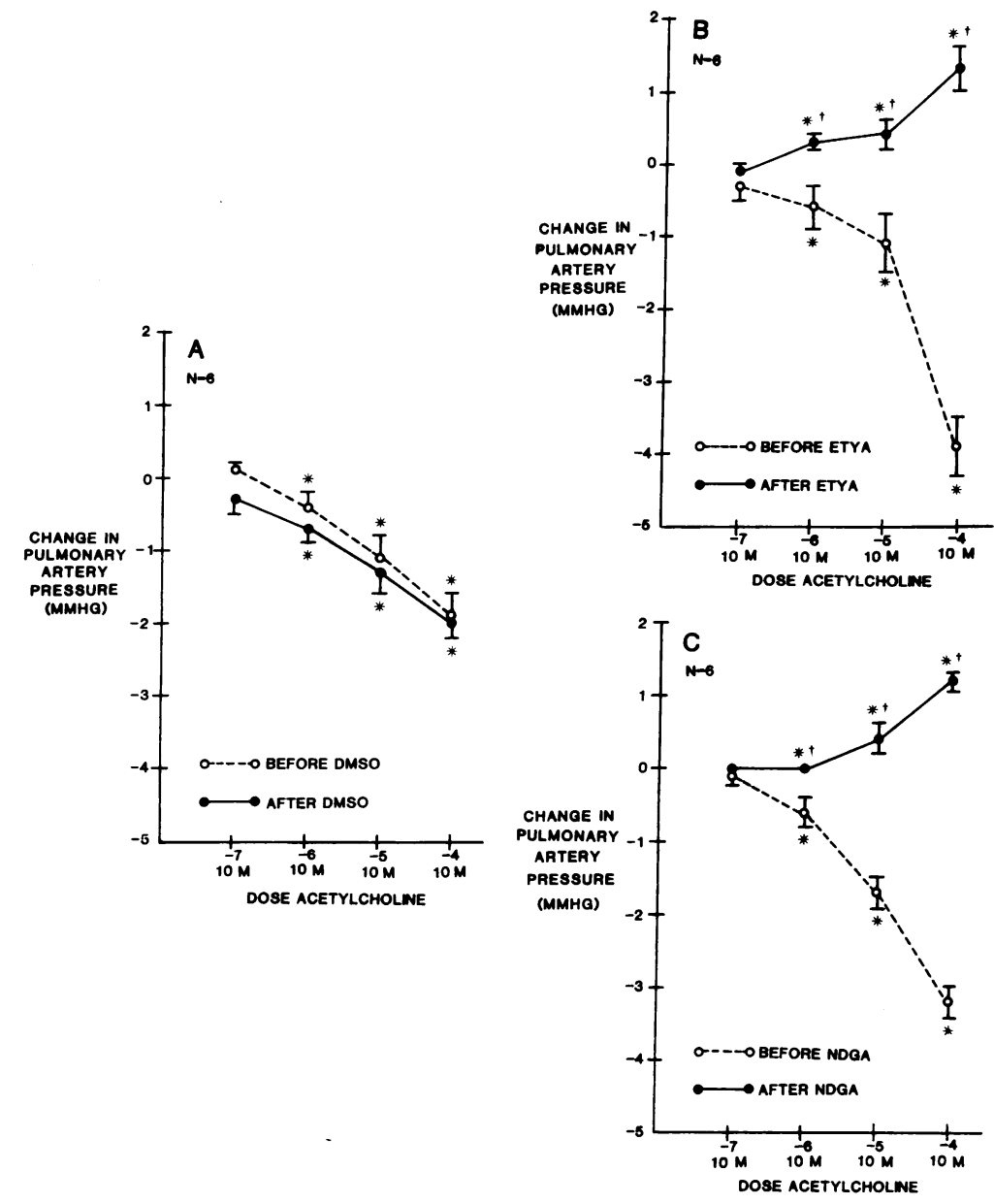

Figure 7. $(A)$ In the presence of NE-induced elevated resistance, the pulmonary arterial vasodilatory response to acetylcholine injection at increasing doses was unchanged by administration of vehicle (DMSO). $\circ$, before DMSO administration; •, after DMSO administration. $(B)$ Administration of the in vitro EDRF inhibitor ETYA $\left(1 \times 10^{-4} \mathrm{M}\right)$ resulted in a loss of the pulmonary arterial vasodilatory response to acetylcholine injection. o, before ETYA administration; •, after ETYA administration. $(C)$ Administration of the in vitro EDRF inhibitor NDGA $\left(1 \times 10^{-4} \mathrm{M}\right)$ resulted in a loss of the pulmonary arterial vasodilatory response to acetylcholine injection. $\circ$, before NDGA administration; $\bullet$, after NDGA administration. *Significantly different from the change in pulmonary artery pressure seen with $10^{-7} \mathrm{M}$ acetylcholine injection, $P<0.05$. 'Significant difference between the change in pulmonary artery pressure seen with the same dose of acetylcholine after ETYA or NDGA administration as compared with after DMSO administration alone, $P<0.01$.

in the presence of an intact endothelium, numerous investigators have identified endothelium-dependent vasodilatory capacity with a large number of substances and in a wide variety of vascular tissue sources (23). The hypothesis was put forth that acetylcholine resulted in the release of a factor(s) that, on diffusing to the subadjacent smooth muscle cells, activated a
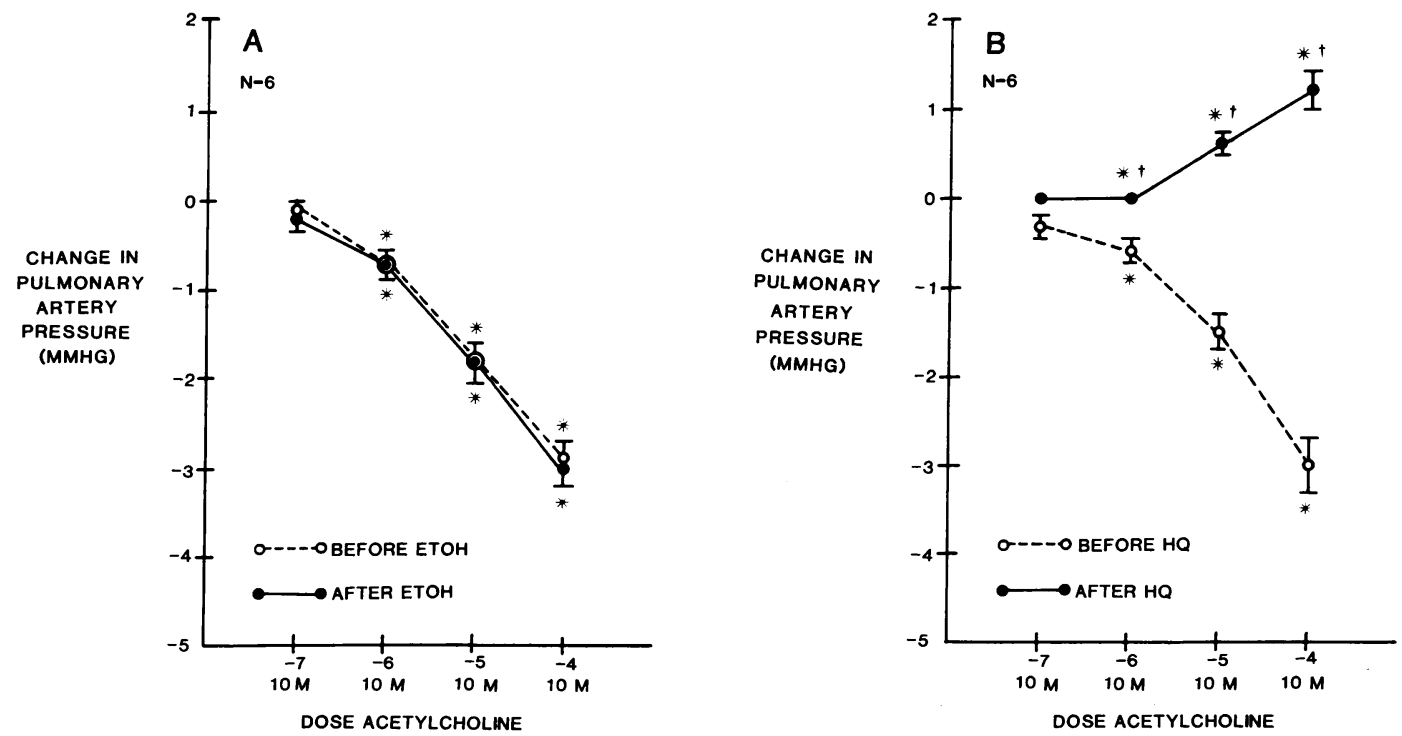

Figure 8. $(A)$ In the presence of NE-induced elevated resistance, the pulmonary arterial vasodilatory response to acetylcholine injection at increasing doses was unchanged by administration of vehicle (ETOH). o, before ETOH administration; •, after ETOH administration. (B) Administration of the in vitro EDRF inhibitor HQ $\left(1 \times 10^{-4} \mathrm{M}\right)$ resulted in a loss of the pulmonary arterial vasodilatory response to acetylcholine injection. $\circ$, before $\mathrm{HQ}$ administration; $\bullet$, after HQ administration. *Significantly different from

the change in pulmonary artery pressure seen with $10^{-7} \mathrm{M}$ acetylcholine injection, $P<0.05$. ${ }^{\dagger}$ Significant difference between the change in pulmonary artery pressure seen with the same dose of acetylcholine after HQ administration as compared with after ETOH administration alone, $P<0.01$. 
mechanism for relaxation (3). Further studies showed that endothelium-dependent relaxation could be restored to strips denuded of endothelium by apposition with, or superfusion from intact endothelium $(3,6,21)$. These studies clearly documented that a diffusible vasodilator was released from the intact endothelium in response to a stimulator. Attempts to identify the nature of this transferable EDRF led investigators to describe a number of substances that blocked endotheliumdependent relaxation in arterial strips without affecting the activity of endothelium-independent vasodilators such as sodium nitroprusside. These in vitro EDRF inhibitors included the lipoxygenase inhibitor ETYA (3), the lipoxygenase inhibitor and antioxidant NDGA (24), and the antioxidant HQ (6). Although the nature of EDRF has never been clearly explained, the activities of these EDRF inhibitors have led investigators to propose a number of possible sources for EDRF, including oxidative products of the lipoxygenase or epoxygenase pathways of arachidonate metabolism (25-27). Further studies showed that pulmonary artery strips had the capacity to respond to endothelium-dependent vasodilators $(7,28)$.

In view of these previous investigations, the results presented here demonstrate endothelium-dependent vasodilation in the pulmonary vascular bed, and are consistent with an important role of the endothelium in the modulation of the hypoxic pressor response of the intact pulmonary vascular bed through the release of EDRF. These observations confirm previous studies that document that the endothelium-dependent vasodilators acetylcholine (29), A23187 (30), and histamine (31) have been shown to dilate the rat pulmonary circulation. Cherry and Gillis (9) observed recently that dose-dependent vasodilatory responses to acetylcholine in precontracted in situ indomethacin-treated rabbit lungs were blocked by administration of the EDRF inhibitors quinacrine and hemoglobin. Moreover, the putative in vitro EDRF inhibitor BW 755C (26) enhanced the pulmonary hypoxic pressor response in intact anesthetized dogs with acute left lower lobe atelectasis (32). Together, the present observations and these previous studies support a role for EDRF in the modulation of pulmonary vascular tone.

The question could be raised whether the EDRF antagonists enhanced hypoxic vasoconstriction through inhibition of basal release of EDRF, or blockade of enhanced EDRF release during hypoxia. As EDRF inhibition during normoxic ventilation did not significantly affect baseline pulmonary artery pressures or the rate of rise in baseline pressures as compared with vehicle controls (Table I), it is unlikely that this augmentation of hypoxic vasoconstriction was due to a decrease in basal EDRF activity, although the possibility of some effect by the inhibitors on basal EDRF release has not been fully excluded. The accentuation of the hypoxic pressor response by EDRF inhibition implies that EDRF activity is increased during hypoxia.

An increase in EDRF activity with hypoxia could be challenged by previous suggestions that anoxia diminished endothelium dependent relaxation responses in vitro $(18,19)$. However, Chand and Altura (7) showed that hypoxia had no effect on acetylcholine-induced endothelium-dependent vasodilation. Recent studies have demonstrated that isolated perfused rat lungs preconstricted by alveolar hypoxia retain the ability to vasodilate in response to acetylcholine (29), A23187 (30), and arachidonate (33). Hypoxic vasoconstriction has been shown to stimulate the release of $\mathrm{PGI}_{2}$ (33) and 5-hydroxy- eicosatetraenoic acid (34), a product of the 5-lipoxygenase pathway of arachidonate metabolism, into the effluent from isolated saline-perfused rat lungs demonstrating that eicosanoid production is stimulated by hypoxic ventilation, including metabolites of arachidonate that may include EDRF. Together, these studies suggest that although anoxia is an inhibitor of EDRF activity, lesser degrees of hypoxia do not inhibit and may stimulate EDRF production and release.

Whereas the specificity of ETYA, NDGA, and HQ as inhibitors of EDRF when used in an intact blood-perfused circulation has never been documented, there is supportive evidence that these agents inhibited endothelium-dependent relaxation in the studies presented here: $(a)$ although the possibility exists with each of these inhibitors of effects independent of EDRF, the consistent enhancement of hypoxic vasoconstriction by three chemically dissimilar antagonists strongly supports a common mechanism, i.e., inhibition of EDRF; (b) we have documented that ETYA, NDGA, and HQ each blocked acetylcholine-induced endothelium-dependent vasodilation in the denervated whole blood-perfused rat lung; and (c) ETYA, NDGA, and HQ have previously been observed to antagonize endothelium-dependent relaxation in numerous studies $(3,6,24)$.

In this study, Ang II vasoconstriction was potentiated by cyclooxygenase inhibition and by the vehicles DMSO and ETOH, but was not further enhanced by the putative EDRF inhibitors ETYA, NDGA, and HQ. The accentuation of Ang II vasoconstriction by cyclooxygenase inhibition in the isolated perfused rat lung has been previously described by Voelkel et al. (33). A previous study by Yilmaz et al. (35) observed heightening of Ang II constriction of rabbit aortic strips by $\mathrm{HQ}$ and denudation of the endothelium, but not by aspirin administration. However, these observations conflict with studies from one of our laboratories, which found no effect on Ang II responsiveness of rabbit thoracic aorta by denudation of the endothelium or by a number of EDRF inhibitors (8). Despite this conflict between our previous studies and the observations by Yilmaz et al., several additional explanations may be considered for the absence of augmentation of Ang II responsiveness by EDRF antagonists in our cyclooxygenase inhibited preparation: $(a)$ there may be differences in reactivity between systemic and pulmonary vessels and between whole blood perfused vascular beds and larger vessels studied in ring preparations; and $(b)$ it is unclear why the vehicles alone heightened Ang II constriction in this study, but may be related to endothelium-independent mechanisms directly involving the vascular smooth muscle (36). Nevertheless, this effect of the vehicles may have masked further potentiation of Ang II vasoconstriction by the putative EDRF antagonists. Unfortunately, we have no direct information from this study on these possibilities.

It is unlikely that the inhibitors enhanced hypoxic pulmonary vasoconstriction through diminished prostacyclin production from cyclooxygenase inhibition, although one of the antagonists, ETYA, is an inhibitor of cyclooxygenase (37). In the experiments presented here, $20 \mu \mathrm{g} / \mathrm{ml}$ meclofenamate was added to the reservoir blood to remove any possible effects by the products of the cyclooxygenase pathway. The early administration of meclofenamate in all protocols resulted in no significant change in the hypoxic pressor response, which is different from previous observations by McMurtry et al. McMurtry et al. (11) previously has shown an increase in hy- 
poxic vasoconstriction after meclofenamate but used a lower dose $(2 \mu \mathrm{g} / \mathrm{ml})$ and administered it later after hypoxic responses had reached a plateau or started to decline. Morganroth et al. (38) used the meclofenamate concentration of 20 $\mu \mathrm{g} / \mathrm{ml}$ to inhibit the cyclooxygenase pathway in the isolated saline-perfused rat lung. Although Voelkel et al. (33) found that a higher meclofenamate dose of $50 \mu \mathrm{g} / \mathrm{ml}$ was required to completely reverse the vasodilatory effects of arachidonate infusion in a blood-perfused lung, administration of less than one tenth of that dose already has been shown to markedly elevate the baseline pulmonary artery pressure in the isolated perfused rat lung (11). As it is highly unlikely that the cyclooxygenase pathway plays an important role in EDRF activity, and because an elevation of the pulmonary artery pressure baseline might significantly affect the results of these experiments, we used a concentration of $20 \mu \mathrm{g} / \mathrm{ml}$ reservoir blood. This dose resulted in an augmentation of the pressor responses to Ang II administration comparable to that which had been seen with the higher dose of meclofenamate in the blood-perfused rat lung (33).

Previous investigators have suggested that hypoxic vasoconstriction is mediated by products of the lipoxygenase pathway of arachidonate metabolism $(34,38)$. The results presented here argue against a significant role for lipoxygenase metabolites in the vasoconstrictor response to hypoxia, as ETYA and NDGA (both lipoxygenase inhibitors) resulted in an accentuation rather than a diminishment of the hypoxic pressor response.

In summary, the results presented in this study demonstrate that the in vitro antagonists of EDRF significantly augment the hypoxic pulmonary pressor response in a denervated but otherwise intact pulmonary vascular bed. This suggests that during hypoxia, the endothelium releases relaxing factor(s) that limit or attenuate the degree of vasoconstriction.

\section{Acknowledgments}

We would like to thank Hoffmann-La Roche Inc. for providing ETYA. The sodium meclofenamate used in this study was kindly provided by Warner-Lambert Co. We wish to thank Ms. Elizabeth Crawford for typing the manuscript.

This work was supported by the Virginia Affiliate, American Heart Association, and by National Institutes of Health research awards HL-28118 and F32 HL-07134.

\section{References}

1. Reeves, J. T., and B. M. Groves. 1984. Approach to the patient with pulmonary hypertension. In Pulmonary Hypertension. E. K. Weir and J. T. Reeves, editors. Futura Publishing Co., Mount Kisco, NY. 1-44.

2. von Euler, U. S., and G. Liljestrand. 1946. Observations on the pulmonary arterial blood pressure in the cat. Acta. Physiol. Scand. 12:301-320.

3. Furchgott, R. F., and J. V. Zawadzki. 1980. The obligatory role of endothelial cells in the relaxation of arterial smooth muscle by acetylcholine. Nature (Lond.). 288:373-376.

4. Peach, M. J., A. L. Loeb, H. A. Singer, and J. Saye. 1985. Endothelium-derived vascular relaxing factor. Hypertension. 7(Suppl. I):I94-I100.

5. Peach, M. J., H. A. Singer, and A. L. Loeb. 1985. Mechanisms of endothelium-dependent vascular smooth muscle relaxation. Biochem. Pharmacol. 34:1867-1874.

6. Griffith, T. M., D. H. Edwards, M. J. Lewis, A. C. Newby, and
A. H. Henderson. 1984. The nature of endothelium-derived vascular relaxant factor. Nature (Lond.). 308:645-647.

7. Chand, N., and B. M. Altura. 1981. Acetylcholine and bradykinin relax intrapulmonary arteries by acting on endothelial cells: role in lung vascular diseases. Science (Wash. DC). 213:1376-1379.

8. Singer, H. A., and M. J. Peach. 1983. Endothelium-dependent relaxation of rabbit aorta. II. Inhibition of relaxation stimulated by methacholine and A23187 with antagonists of arachidonic acid metabolism. J. Pharmacol. Exp. Ther. 226:796-801.

9. Cherry, P. D., and C. N. Gillis. 1987. Evidence for the role of endothelium-derived relaxing factor in acetylcholine-induced vasodilation in the intact lung. J. Pharmacol. Exp. Ther. 241:516-520.

10. Hauge, A. 1968. Role of histamine in hypoxic pulmonary hypertension in the rat. I. Blockade or potentiation of endogenous amines, kinins, and ATP. Circ. Res. 22:371-383.

11. McMurtry, I. F., A. B. Davidson, J. T. Reeves, and R. F. Grover. 1976. Inhibition of hypoxic pulmonary vasoconstriction by calcium antagonists in isolated rat lungs. Circ. Res. 38:99-104.

12. Rounds, S. and I. F. McMurtry. 1981. Inhibitors of oxidative ATP production cause transient vasoconstriction and block subsequent pressor responses in rat lungs. Circ. Res. 48:393-400.

13. Duff, F., A. D. Greenfield, J. T. Shepherd, and I. D. Thompson. 1953. A quantitative study of the response to acetylcholine and histamine of the blood vessels of the human hand and forearm. J. Physiol. (Lond.). 120:160-170.

14. Steel, R. G. D., and J. H. Torrie. 1980. Principles and Procedures of Statistics. McGraw-Hill Book Co., New York. 137-238.

15. Miller, M. A., and C. A. Hales. 1979. Role of cytochrome P-450 in alveolar hypoxic pulmonary vasoconstriction in dogs. J. Clin. Invest. 64:666-673.

16. Fishman, A. P. 1976. Hypoxia on the pulmonary circulation. How and where it acts. Circ. Res. 38:221-231.

17. Bergofsky, E. H., and S. Holtzman. 1967. A study of the mechanisms involved in the pulmonary arterial pressor response to hypoxia. Circ. Res. 20:506-519.

18. De Mey, J. G., and P. M. Vanhoutte. 1981. Contribution of the endothelium to the response to anoxia in the canine femoral artery. Arch. Int. Pharmacodyn. 253:325-326.

19. De Mey, J. G. and P. M. Vanhoutte. 1982. Heterogeneous behavior of the canine arterial and venous wall. Circ. Res. 51:439-447.

20. Holden, W. E., and E. McCall. 1984. Hypoxia-induced contractions of porcine pulmonary artery strips depend on intact endothelium. Exp. Lung. Res. 7:101-112.

21. Rubanyi, G. M., and P. M. Vanhoutte. 1985. Hypoxia releases a vasoconstrictor substance from the canine vascular endothelium. $J$. Physiol. (Lond.). 364:45-56.

22. Hickey, K. A., G. Rubanyi, R. J. Paul, and R. F. Highsmith. 1985. Characterization of a coronary vasoconstrictor produced by cultured endothelial cells. Am. J. Physiol. 248:C550-C556.

23. Furchgott, R. F. 1984. The role of endothelium in the responses of vascular smooth muscle to drugs. Annu. Rev. Pharmacol. Toxicol. 24:175-97.

24. Singer, H. A., and M. J. Peach. 1983. Endothelium-dependent relaxation of rabbit aorta. I. Relaxation stimulated by arachidonic acid. J. Pharmacol. Exp. Ther. 226:790-795.

25. Forstermann, U., and B. Neufang. 1984. The endothelium-dependent relaxation of rabbit aorta: effects of antioxidants and hydroxylated eicosatetraenoic acids. Br. J. Pharmacol. 82:765-767.

26. Forstermann, U., and B. Neufang. 1984. The endothelium-dependent vasodilator effect of acetylcholine: characterization of the endothelial relaxing factor with inhibitors of arachidonic acid metabolism. Eur. J. Pharmacol. 103:65-70.

27. Vanhoutte, P. M. 1987. The end of the quest? Nature (Lond.). 327:459-460.

28. Ignarro, L. J., T. M. Burke, K. S. Wood, M. S. Wolin, and P. J. Kadowitz. 1984. Association between cyclic GMP accumulation and acetylcholine-elicited relaxation of bovine intrapulmonary artery. $J$. Pharmacol. Exp. Ther. 228:682-690. 
29. Feddersen, C. O., M. M. Mathias, I. F. McMurtry, and N. F. Voelkel. 1986. Acetylcholine induces vasodilatation and prostacyclin synthesis in rat lungs. Prostaglandins. 31:973-987.

30. McMurtry, I. F. 1985. Bay K 8644 potentiates and A23187 inhibits hypoxic vasoconstriction in rat lungs. Am. J. Physiol. 249:H741-H746.

31. Thompson, B., G. R. Barer, and J. W. Shaw. 1976. The action of histamine on pulmonary vessels of cats and rats. Clin. Exp. Pharmacol. Physiol. 3:399-414.

32. Garrett, R. C., S. Foster, and H. M. Thomas III. 1987. Lipoxygenase and cyclooxygenase blockade by BW $755 \mathrm{C}$ enhances pulmonary hypoxic vasoconstriction. J. Appl. Physiol. 62:129-133.

33. Voelkel, N. F., J. G. Gerber, I. F. McMurtry, A. S. Nies, and J. T. Reeves. 1981. Release of vasodilator prostaglandin, $\mathbf{P G I}_{2}$, from isolated rat lung during vasoconstriction. Circ. Res. 48:207-213.
34. Voelkel, N. F., M. Morganroth, and C. O. Feddersen. 1985. Potential role of arachidonic acid metabolites in hypoxic pulmonary vasoconstriction. Chest. 88:245S-248S.

35. Yilmaz, G., H. E. Aksulu, E. Demirel, Z. S. Ercan, H. Zengil, and R. K. Turker. 1987. Modulation by endothelium of the vascular effects of angiotensin II. Agents Actions. 21:184-190.

36. Gruetter, C. A., E. T. Ryan, and D. D. Schoepp. 1987. Endothelium enhances tachyphylaxis to angiotensins II and III in rat aorta. Eur. J. Pharmacol. 143:139-142.

37. Ahern, D. G., and D. T. Downing. 1970. Inhibition of prostaglandin biosynthesis by eicosa-5,8,11,14-tetraynoic acid. Biochim. Biophys. Acta. 210:456-461.

38. Morganroth, M. L., J. T. Reeves, R. C. Murphy, and N. F. Voelkel. 1984. Leukotriene synthesis and receptor blockers block hypoxic pulmonary vasoconstriction. J. Appl. Physiol. 56:1340-1346. 